\title{
Electron-electron interactions and topology in the electronic properties of gated graphene nanoribbon rings in Möbius and cylindrical configurations
}

\author{
Alev Devrim Güçlï, ${ }^{1,2}$ Marek Grabowski, ${ }^{3}$ and Pawel Hawrylak ${ }^{1}$ \\ ${ }^{1}$ Quantum Theory Group, Security and Disruptive Technology, Emerging Technologies Division, National Research Council of Canada, \\ Ottawa, Canada and Department of Physics, University of Ottawa, Ottawa, Canada \\ ${ }^{2}$ Department of Physics, Izmir Institute of Technology, IZTECH, TR35430, Izmir, Turkey \\ ${ }^{3}$ Department of Physics, University of Colorado at Colorado Springs, Colorado Springs, Colorado 80918, USA
}

(Received 21 November 2012; published 30 January 2013)

\begin{abstract}
We present a theory of the electronic properties of gated graphene nanoribbon rings with zigzag edges in Möbius and cylindrical configurations. The finite width opens a gap and nontrivial topology of the Möbius ring leads to a single edge with edge states with an induced, effective gauge field, in analogy to topological insulators. The single zigzag edge leads to a shell of degenerate states at the Fermi level and a ferromagnetic (FM) ground state at half-filling, i.e., at charge neutrality, due to electron-electron interactions. For fractional fillings, both the magnetic moment and the energy gap are found to oscillate as a function of the shell filling. In cylindrical rings, the two edges lead to AF ground state at half-filling but FM ground state at fractional fillings.
\end{abstract}

DOI: 10.1103/PhysRevB.87.035435

PACS number(s): 73.22.-f, 73.22.Pr

\section{INTRODUCTION}

Graphene, ${ }^{1-5}$ a single layer of graphite, shows promise as a material for nanoelectronics due to high electronic and thermal conductivity. As a result, there is increasing interest in understanding properties of graphene engineered at the nanoscale to form graphene nanoribbons and dots. Graphene nanoribbons are quasi-one-dimensional structures ${ }^{6-37}$ with electronic properties depending on the angle at which they are cut. This includes a tunable energy gap with semimetallic or semiconductor behavior, ${ }^{13-15}$ one-dimensional edge states at the Fermi level with unusual magnetic properties with possible spintronic applications, ${ }^{16-23}$ and solitonic edge states as in polyacetylene. ${ }^{37-39}$

Graphene nanoribbons also played an important role in inspiring the field of topological insulators. ${ }^{6-12}$ The interior of the graphene ribbon acts like an insulator with a gap in the energy spectrum, whereas the energies of the edge states are in the middle of the gap. The topological aspect of the edge states is generated by the spin-orbit coupling which lifts the spin degeneracy at a given edge, leading to graphene nanoribbon as a spin Hall insulator. ${ }^{6}$ Unfortunately, spin-orbit coupling in graphene is found to be too small to give rise to a spin Hall effect. ${ }^{10}$ Interestingly, it has been suggested that nontrivial properties of graphene nanoribbons can be generated directly by engineering a nontrivial Möbius geometry of the nanoribbon without the need for the spin-orbit coupling. ${ }^{40-51}$ For example, it was shown by Guo et al. ${ }^{46}$ that one electron states of a class of Möbius graphene ribbons with zigzag edges can be understood by introducing a non-Abelian gauge field ${ }^{46}$ as in topological insulators. ${ }^{6-12}$ However, these authors used the $s$ orbitals instead of $p_{z}$ orbitals as the basis needed to describe graphene nanoribbons and limited their work to an even number of atomic chains. The effects of Coulomb interactions on the magnetic structure of zigzag graphene nanoribbons with an even number of carbon chains were studied within mean-field Hubbard models ${ }^{42,43}$ and density functional theories. ${ }^{44,47}$ It was found that mean-field Hubbard treatment of Coulomb interactions gives a minimal spin polarization and an associated magnetic domain wall, ${ }^{42,43}$ whereas density functional calculations predict finite total magnetization. ${ }^{44,47}$ On the experimental side, although we are not aware of Möbius graphene nanoribbons with zigzag edges yet, twisted nanoribbons were recently obtained inside carbon nanotubes through electron irradiation of functionalized fullerenes $^{36}$ and a stable Möbius aromatic hydrocarbon was synthesized by Ajami et $a l^{41}$

In this work we describe the effect of Möbius topology on the one-electron properties of graphene nanoribbon rings as a function of the number of carbon chains, both even and odd. We compare the Möbius topological insulator with normal insulators, cylindrical graphene nanoribbon rings with cyclic boundary conditions. Our main contribution involves inclusion of electron-electron interaction using configuration-interaction method (CI) combined with tight-binding and Hartree-Fock approach in the Möbius topological insulator with carrier density controlled by an external gate.

We show that the magnetic properties of the nanoribbons are strongly affected by the filling factor of the edge states due to degeneracies and electron-electron interactions. The dependence of the magnetization on the filling factor is drastically different for Möbius ribbons with even and odd number of chains, and cyclic ribbons. Away from charge neutrality, strong oscillations of edge magnetization as a function of filling factor are observed for Möbius ribbons. The cyclic nanoribbon is found to be antiferromagnetic at halffilling but becomes ferromagnetic away from charge neutrality. The magnetic properties of a Möbius ribbon have similarities with triangular graphene quantum dots with zigzag edges. ${ }^{52-54}$ Indeed, both systems have only a single edge and insulating bulk, and we compare the magnetic properties as a function of the filling of the edge states with carriers in both structures.

The paper is organized as follows. In Sec. II we describe single particle properties in the tight-binding approximation. We review basic properties of single and double chain nanoribbons with Möbius and cyclic configurations. Then we derive analytical and semianalytical results for larger nanoribbon rings with even and odd number of chains. In Sec. III we use a tight-binding-Hartree-Fock-configurationinteraction method to investigate many-body properties of 
Möbius and cyclic nanoribbon rings as a function of filling factor of edge states. Summary and conclusion are contained in Sec. IV.

\section{SINGLE PARTICLE PROPERTIES OF GRAPHENE NANORIBBON RINGS IN MÖBIUS AND CYLINDRICAL CONFIGURATIONS}

In this section we review the tight-binding method applied to the single particle properties of graphene with standard periodic and Möbius boundary conditions. For charge neutral nanoribbon, there is one $p_{z}$ electron for every carbon atom. The remaining three valence electrons occupy the $s, p_{x}$, and $p_{y}$ orbitals to form $s p^{2}$ bonds with the three nearest in-plane neighbors. The $s p^{2}$ bond electrons are responsible for the structural properties of the system and are neglected in the study of the electronic properties determined by the $p_{z}$ orbitals.

The one electron states of graphene nanoribbon can be written as a linear combination of $p_{z}$ orbitals on every carbon atom. The resulting tight-binding Hamiltonian ${ }^{1}$ is given by

$$
H=\sum_{i, l, \sigma} t_{i l \sigma} c_{i \sigma}^{\dagger} c_{l \sigma},
$$

where the operator $c_{i \sigma}^{\dagger}$ creates an electron on the $i$ th $p_{z}$ orbital with spin $\sigma, t_{i l \sigma}$ is an effective hopping integral which describes the probability of scattering of electron from the $l$ th $p_{z}$ orbital $\phi_{l}$ to the $i$ th $p_{z}$ orbital $\phi_{i}$.

Before discussing the single particle properties of graphene nanorings with zigzag edges, we would like to point out that the stability of zigzag edges in graphene nanostructures is an active research subject. ${ }^{55-61}$ Theoretical calculations ${ }^{55,56,59,60}$ show that if the dangling bonds on the zigzag edges are passivated by a single hydrogen each, then a stable zigzag edge can be obtained. Otherwise an edge reconstruction may occur. The stability of zigzag edges free of reconstruction was recently confirmed experimentally. ${ }^{61}$

\section{A. Heilbronner ring}

The simplest Möbius system of $\pi$ electrons is a Möbius annulene first studied by Heilbronner. ${ }^{48}$ Heilbronner considered a ring of carbon atoms with $p_{z}$ orbitals rotating by a constant amount when moving from atom to atom around the ring, as shown in Fig. 1(a). The ring can also be viewed as a cyclic polyacetylene with Möbius boundary condition. Within this model, starting with atom at 0 , the hopping integral between neighboring atoms $t$ should be replaced by $t_{M}=t \cos (\pi / M)$, where $M$ is the number of atoms in the ring. This substitution fails if we want to tunnel from the last $M-1$ orbital to the first 0th orbital. This is seen in Fig. 1 where the signs of the $p_{z}$ wave functions are shown in dark gray (blue color online) and light gray (red color online), showing that at the point where atom number 0 meets atom number $M-1$ the hopping matrix element becomes $-t \cos (\pi / M)$ due to the opposite orientation of $p_{z}$ orbitals. The change of sign of the tunneling matrix element is possible because of the internal degree of freedom of each carbon atom-direction of the quantization axis. The energy spectra for the Möbius and cyclic annulene

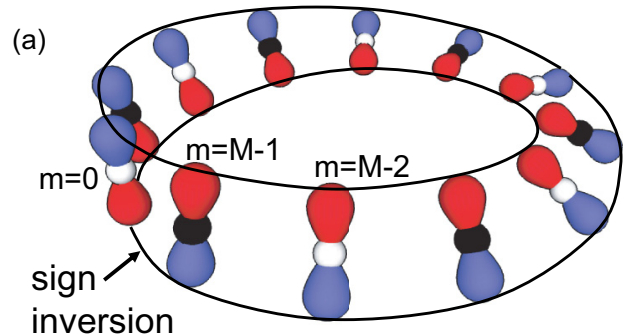

(b)

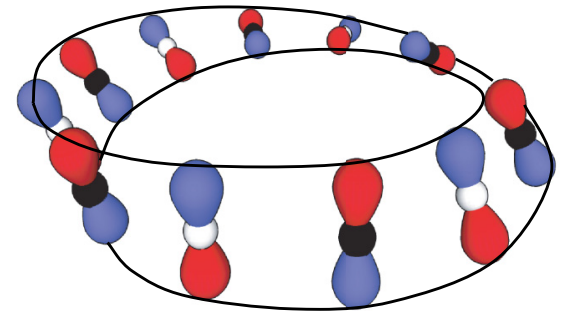

FIG. 1. (Color online) (a) Heilbronner ring with $M p_{z}$ orbitals. Different gray scale (blue and red colors online) represent sign of the wave function. The Möbius condition requires a sign inversion between the 0 th and $M-1$ th sites. (b) For a Heilbronner ring with odd $M, p_{z}$ orbitals can be chosen to be alternating their sign from site to site.

are then given by Heilbronner in Ref. 48:

$$
\begin{aligned}
E_{j}^{\mathrm{cyc}} & =2 t_{M} \cos \left(\frac{2 j \pi}{M}\right), \\
j & =0,1, \ldots, M-1(M=\text { even or odd }), \\
E_{j}^{\mathrm{Mob}} & =2 t_{M} \cos \left[\frac{(2 j+1) \pi}{M}\right], \\
j & =0,1, \ldots, M-1(M=\text { even or odd }) .
\end{aligned}
$$

The energy spectra can be obtained using periodicity condition $\psi_{m+M}=\psi_{m}$ for the cyclic annulene, and $\psi_{m+M}=-\psi_{m}$ for the Möbius annulene. Alternatively, the basis set of $p_{z}$ orbitals can be chosen in such a way that $p_{z}$ orbitals alternate their sign, as shown in Fig. 1(b). As we will see later, this choice can be practical for more complex structures. For a Heilbronner ring with odd $M$, we then need to replace $t_{M}$ by $-t_{M}$ and we no longer need to change the sign of the hopping parameter between atoms 0 and $M-1$. Thus, we can use periodic boundary condition $\psi_{m+M}=\psi_{m}$ with reversed overall sign of the hopping parameter to obtain

$$
\begin{aligned}
E_{j}^{\mathrm{Mob}} & =-2 t_{M} \cos \left[\frac{(2 j+1) \pi}{M}\right], \\
j & =0,1, \ldots, M-1(M=\text { odd }) .
\end{aligned}
$$

Hence, the energy spectrum of a Heilbronner Möbius ring with odd $M$ is the mirror image of the energy spectrum of a cyclic ring, if normalized by their respective effective hopping parameter $t_{M}$. In the following we will ignore the $M$ dependence of the hopping parameter and take $t_{M}=$ $t \cos (\pi / M) \approx t$ for large $M$.

\section{B. Polyacene ring}

The narrowest graphene nanoribbons can be formed by bringing together two chains of polyacetylene, as seen in Fig. 2. 
(a)

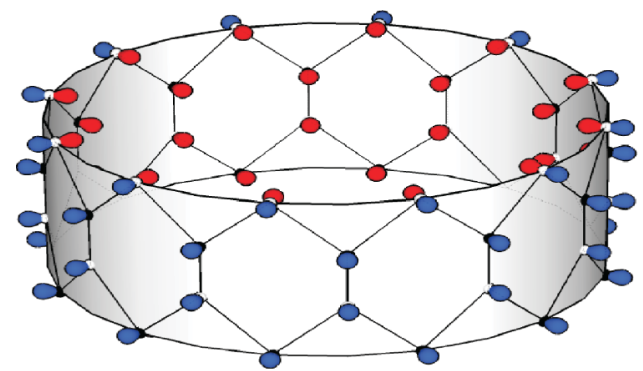

(b)

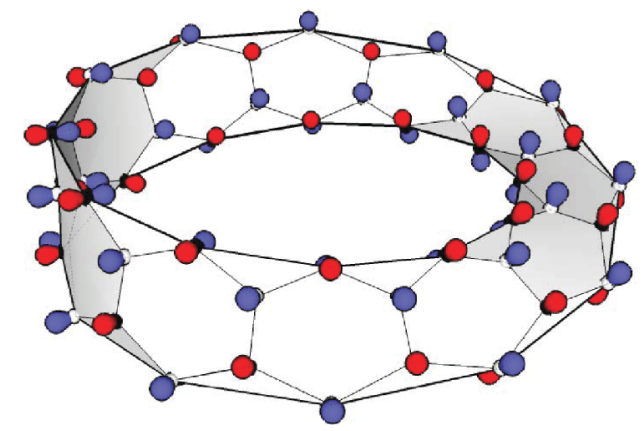

FIG. 2. (Color online) Polyacene ring with (a) cyclic and (b) Möbius periodicity. In the Möbius polyacene ring, $p_{z}$ orbitals are chosen to be alternating their sign from site to site.

Such structures are also called polyacene. In polyacene rings, cyclic [Fig. 2(a)] or Möbius [Fig. 2(b)], the number of atoms in a chain $M$ must be even in order to keep the hexagonal structure intact. The analytical solutions to the cyclic and Möbius structures were previously calculated, ${ }^{49,50}$ and are given by

$$
\begin{aligned}
E_{j}^{\mathrm{cyc}} & = \pm \frac{t}{2}\left[1 \pm \sqrt{1+16 \cos ^{2}\left(\frac{2 j \pi}{M}\right)}\right], \\
j & =0,1, \ldots, M / 2-1(M=\text { even }), \\
E_{j}^{\mathrm{Mob}} & =\frac{t}{2}\left[(-1)^{j} \pm \sqrt{1+16 \cos ^{2}\left(\frac{j \pi}{M}\right)}\right], \\
j & =0,1, \ldots, M-1(M=\text { even }) .
\end{aligned}
$$

Note that, in each case, the total number of hexagons in these structures is given by $M / 2$. Detailed discussion of polyacene rings can be found in Refs. 49 and 50.

\section{Graphene nanoribbon ring with three chains}

We will now derive the secular determinant leading to the analytical energy spectrum for a graphene nanoribbon with three polyacetylene chains, as shown in Fig. 3. Each chain contains $M$ atoms, and the unit cells are defined such that there are a total of $M$ unit cells in the system. There is a condition on the parity of $M$ depending on the boundary condition. In order to keep the hexagonal structure intact, $M$ must be even for a cyclic ring and odd for a Möbius ring.

Although it is not possible to define $A$ and $B$ sublattices in a Möbius ring since the system is not bipartite, for the following analysis we define $A$ and $B$ atoms, shown in light gray (red color online) and dark gray (blue color online). For a Möbius configuration, two atoms of the same type become neighbors at

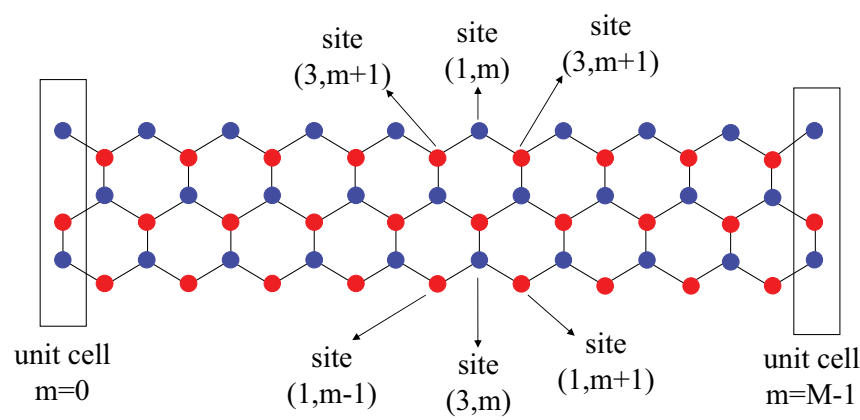

FIG. 3. (Color online) Graphene nanoribbon with three polyacetylene chains. $p_{z}$ orbitals are chosen to be alternating their sign from site to site, shown by different gray scales (red and blue colors online). In any given unit cell, the edge site that has only two neighbors is assigned to be the first site of the unit cell.

the boundary between cell 0 and cell $M-1$. Next, we assign $p_{z}$ orbitals to $A$ and $B$ atoms with opposite polarity for the Möbius ring. The effect of this polarity alternation is that there is an overall change of sign of the hopping parameter $t$ but the boundary condition requires no more special treatment for the change of phase of the hopping matrix element.

The key step is the definition of the $m$ th unit cell creation operator $\psi_{m}^{\dagger}$ as

$$
\psi_{m}^{\dagger}=\left(\begin{array}{c}
c_{1 m}^{\dagger} \\
c_{2 m}^{\dagger} \\
c_{3 m}^{\dagger}
\end{array}\right)
$$

Here the $c_{i m}^{\dagger}$ are defined in a special way. The $c_{1 m}^{\dagger}$ is defined as the creation operator of the edge site that has only two neighbors, $c_{2 m}^{\dagger}$ is for the middle site, and $c_{3 m}^{\dagger}$ is for the remaining site. Thus the order of site operators in $\psi_{m}^{\dagger}$ alternates from cell to cell, as shown in Fig. 3. The advantage of this choice is that we can apply a periodic boundary condition and define the Fourier transform $\psi_{k}$ :

$$
\psi_{m}=\psi_{m+M}=\frac{1}{\sqrt{M}} \sum_{k=0}^{M-1} \psi_{k} e^{-i 2 \pi k m / M} .
$$

The tight-binding Hamiltonian can now be written as

$$
H=-t \sum_{m}\left[\psi_{m}^{\dagger} U \psi_{m}+\left(\psi_{m}^{\dagger} T \psi_{m+1}+\text { H.c. }\right)\right],
$$

where the matrix $U$ describes the hopping terms between sites within a cell $m$, whereas the matrix $T$ describes the hopping terms between cell $m$ and cell $m+1$. The $U$ and $T$ matrices are given by

$$
U=\left(\begin{array}{lll}
0 & 0 & 0 \\
0 & 0 & 1 \\
0 & 1 & 0
\end{array}\right)
$$

and

$$
T=\left(\begin{array}{lll}
0 & 0 & 1 \\
0 & 1 & 0 \\
1 & 0 & 0
\end{array}\right)
$$


Using Eq. (8) we obtain

$$
H=-t \sum_{k} \psi_{k}^{\dagger}\left[U+\left(T e^{i 2 \pi k / M}+\text { H.c. }\right)\right] \psi_{k},
$$

which is diagonal in $k$. The band structure of graphene Möbius ribbon for a given $M$ and $k$ can then be analytically obtained by solving the following secular determinant:

$$
\left|\begin{array}{ccc}
-E & 0 & -2 t \cos \left(\frac{2 \pi k}{M}\right) \\
0 & -2 t \cos \left(\frac{2 \pi k}{M}\right)-E & -t \\
-2 t \cos \left(\frac{2 \pi k}{M}\right) & -t & -E
\end{array}\right|=0,
$$

where $k=0, \ldots, M-1$ and $M$ is odd.

It is important to note that the same equation also applies to a cyclic ring with even $M$. In this case, we no longer need to assign alternating signs to the polarity of $A$ and $B$ atomic orbitals. Thus, the overall sign of $t$ must be reversed in Eq. (13) in order to get correct solutions for a cyclic ring with even $M$.

\section{Graphene nanoribbon ring with $N$ chains}

For odd $N$, the solutions found in the previous subsection are easily generalized to any Möbius ring with odd $M$ or any cyclic ring with even $M$. The $U$ and $T$ matrices of size $N$ are given by

$$
U=\left(\begin{array}{cccccc}
0 & 0 & 0 & 0 & 0 & \ldots \\
0 & 0 & 1 & 0 & 0 & \\
0 & 1 & 0 & 0 & 0 & \\
0 & 0 & 0 & 0 & 1 & \\
0 & 0 & 0 & 1 & 0 & \\
\ldots & & & & & \ldots
\end{array}\right)
$$

and

$$
T=\left(\begin{array}{ccccc}
0 & 0 & 0 & 0 & 1 \\
0 & 0 & 0 & 1 & 0 \\
0 & 0 & \cdots & 0 & 0 \\
0 & 1 & 0 & 0 & 0 \\
1 & 0 & 0 & 0 & 0
\end{array}\right)
$$

The band structure for a given $N, M$, and $k$ can then be calculated by diagonalizing $U+\left(T e^{i 2 \pi k / M}+\right.$ H.c. $)$.

For even $N$, the solutions were analyzed in detail in Ref. 46 for $s$-type orbitals using a bonding and antibonding combination of opposite edge sites of the ribbon. For $p_{z}$ type orbital, the solutions found in Ref. 46 still apply, but with reversed sign of the hopping parameter $t$ since one needs to consider alternating orbital signs for $A$ and $B$ atoms, as discussed earlier. Hence the valence states obtained in this work are conduction states in Ref. 46.

\section{E. Single particle energy spectra of Möbius and cyclic ribbons}

In this subsection we analyze energy spectra of Möbius and cyclic rings as a function of size and parity. In Fig. 4 we focus on even $N$ rings. Figure 4 shows the energy spectrum for the $N=2, M=26$ ring, in cyclic and Möbius configurations, calculated using Eqs. (5) and (6) with $|t|=2.8 \mathrm{eV}$. Since the total number of electrons is equal to the number of sites, we have $N_{e}=52$ electrons filling the first 26 valence levels, assumed for now to be doubly occupied. Note that, close to the

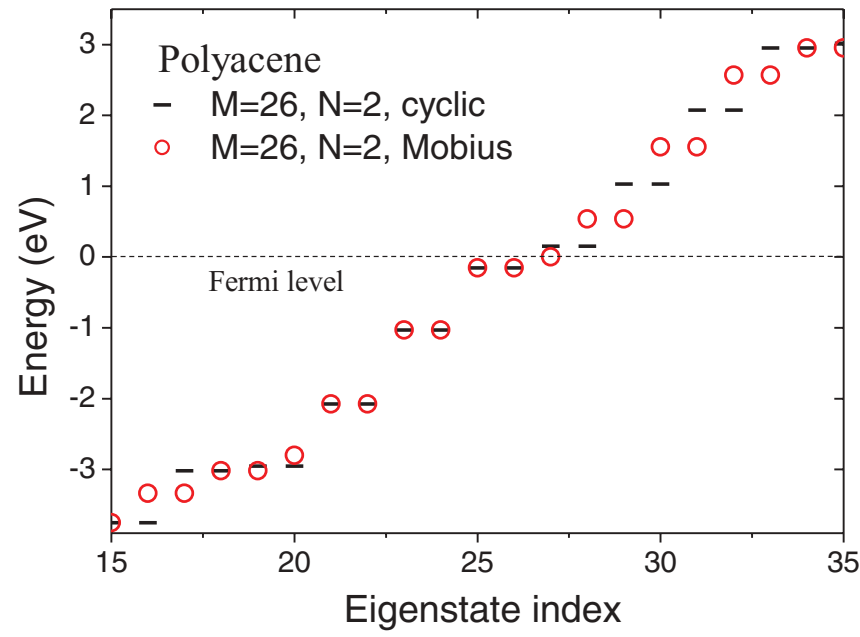

FIG. 4. (Color online) Single particle energy spectrum of polyacene ring with $N=2, M=26$ (total of $N_{a}=52$ atoms), in cyclic (solid line) and Möbius (circles) configurations. Cyclic and Möbius configurations share the same valence band edge state energy levels.

Fermi level, cyclic and Möbius configurations share the same valence levels corresponding to edge states. However, a cyclic configuration has electron-hole symmetry [clearly displayed in Eq. (5)], which is absent in the Möbius configuration [cf. Eq. (6)].

This broken electron-hole symmetry in the Möbius configuration has a subtle but important implication for wide ribbons. Figure 5 is a similar graph to Fig. 4 but for a wider ribbon with $N=14$. For a ribbon with length $M$, as the width $N$ increases, edge states become more distinguishable in the energy spectrum; their energies become increasingly degenerate at the Fermi level, with a substantial energy gap separating them from remaining valence and conduction band levels.

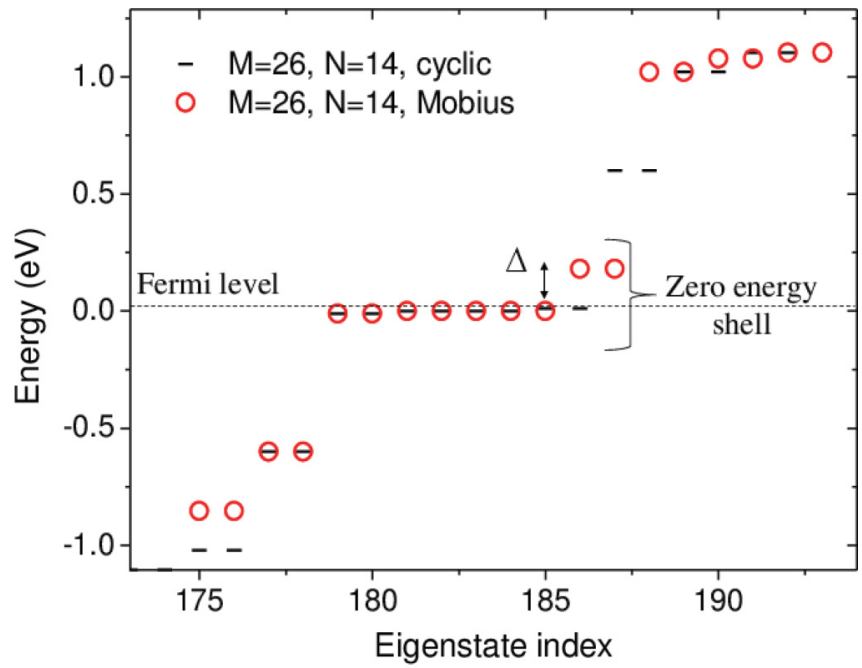

FIG. 5. (Color online) Single particle energy spectrum of nanoribbon ring with $N=14, M=26$ (total of $N_{a}=364$ atoms), in cyclic (solid line) and Möbius (circles) configurations. The Möbius configuration (circles) has nine degenerate edge states occupied by eight electrons. 
The number of edge states can be estimated in the limit of large $N$ and zero energy, following the energy spectra derived in Ref. 46. For the cyclic ring, edge state $k$ values are given by $M / 6<k_{d}<M / 3$ both for valence and conduction spectra. For the Möbius ring we must have $M / 6<k_{d}<M / 3$ for the valence spectrum and $M / 6<k_{d}-1 / 2<M / 3$ for the conduction spectrum. Hence, the total number of edge states is (for a given $M$ ) by one larger in Möbius than cyclic configurations. Figure 5 confirms this assertion. There exists a degenerate shell in the vicinity of the Fermi level (circles), split into a subshell with seven states and a subshell at higher energy $\Delta$ with two states, with a total of nine states, one more than a cyclic ribbon with the same number of atoms, shown as bars in Fig. 5. The splitting of the degenerate shell $\Delta$ is related to the width of the ribbon and decreases with increasing ribbon width. Next, we need to calculate the number of electrons occupying the edge states. Assuming all remaining valence states are doubly occupied, for a ribbon ring in cyclic configuration with a degenerate band of $N_{d}$ edge states, charge neutrality requires the degenerate band to be occupied with $N_{d}$ electrons, leading to antiferromagnetic edge states. ${ }^{42,43}$ In the example given in Fig. 5 for the the cyclic ring, eight electrons occupy eight edge states giving a filling factor $v=1$. The remaining electrons doubly occupy the valence states. However, for the Möbius configuration, an unusual situation occurs. Due to the broken electron-hole symmetry, we have nine degenerate edge states (Fig. 5) occupied by eight electrons, thus filling factor $v \neq 1$. As we will see below, the difference in the filling factor gives rise to different magnetic properties when electron-electron interactions are taken into account.

In Fig. 6 we show the energy spectrum of a wide ribbon with odd number of chains $N$ in Möbius configuration, with $N=13$ and $M=25$. In this case we have nine electrons filling eight edge states, once again leading to $v \neq 1$ as in the even $N$ Möbius ring. However, there is an important difference between the odd $N$ and even $N$ Möbius configurations. Although both systems have a single edge due to the topology, odd $N$ configurations always have an odd number of edge

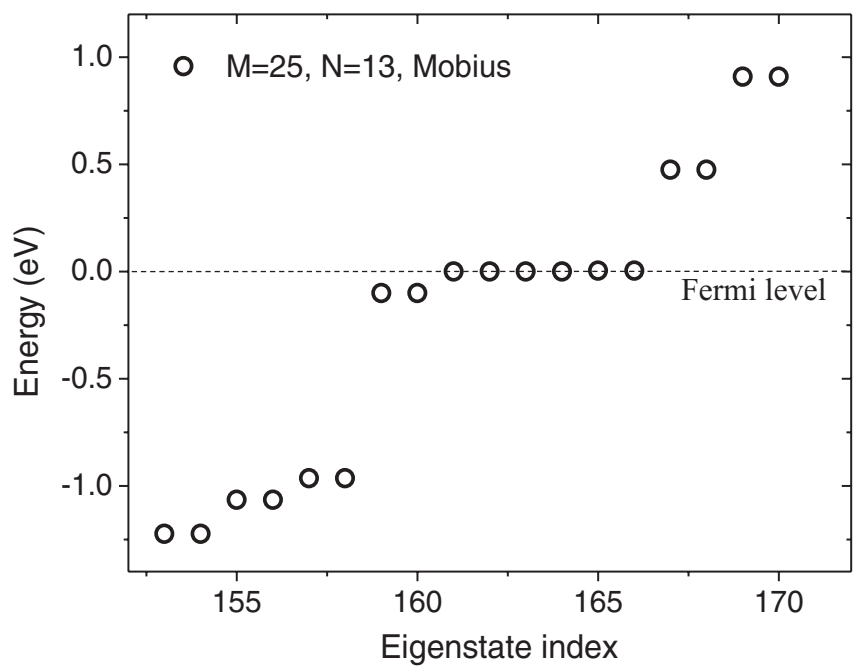

FIG. 6. Single particle energy spectrum of a nanoribbon ring with $N=13, M=25$. Only Möbius configuration is allowed. Eight degenerate edge states are occupied by nine electrons. atoms, whereas even $N$ configurations always have an even number of edge atoms. Indeed, we will see later that the two configurations have significantly different magnetic properties of their edges.

\section{EFFECT OF ELECTRON-ELECTRON INTERACTIONS ON THE ELECTRONIC PROPERTIES OF MÖBIUS AND PERIODIC RINGS}

In systems with high degeneracy of energy levels manybody effects play an important role, with electronic properties determined by electron-electron interactions alone. In order to investigate many-body effects as a function of filling factor of edge states, we use a tight-binding-Hartree-Fockconfiguration interaction (TB-HF-CI) approach. ${ }^{52-54}$ In practice, one needs to apply an external gate potential in order to control the number of electrons in the system. In ring geometries considered here, the electrical field of the gate will create potential gradient across the structure depending on the geometry of the gate and of the ribbon, possibly resulting in a charge density redistribution. Such effects are ignored in our analysis and will need to be investigated for specific experimental realizations of graphene ribbons.

As a first step, we remove electrons from the edge states, and numerically find solutions of the Hartree-Fock Hamiltonian $H_{\mathrm{HF}}$ describing the remaining valence electrons for which a mean-field approach is expected to be sufficient:

$$
\begin{aligned}
H_{\mathrm{HF}}= & \sum_{i j \sigma} \tau_{i j} c_{i \sigma}^{\dagger} c_{j \sigma}+\sum_{i l \sigma} \sum_{j k \sigma^{\prime}}\left(\rho_{j k \sigma^{\prime}}-\rho_{j k \sigma^{\prime}}^{\mathrm{bulk}}\right) \\
& \times\left(\left\langle i j\left|V_{e e}\right| k l\right\rangle-\left\langle i j\left|V_{e e}\right| l k\right\rangle \delta_{\sigma, \sigma^{\prime}}\right) c_{i \sigma}^{\dagger} c_{l \sigma} .
\end{aligned}
$$

We have also included the next-nearest neighbor hopping $t_{n n n}=-0.1 \mathrm{eV}$, in addition to the nearest neighbor hopping $t_{n n}=-2.8 \mathrm{eV}$ in our numerical calculations. The terms $\rho$ and $\rho^{\text {bulk }}$ are nanoribbon and bulk density matrices, respectively. ${ }^{52-54}$ The two-body Coulomb matrix elements $\left\langle i j\left|V_{e e}\right| k l\right\rangle$ computed using Slater $p_{z}$ orbitals include on-site interactions, all scattering and exchange terms within nextnearest neighbors, and all long range direct interactions. It is important to remember that, for Möbius structures, we use alternating signs for the $p_{z}$ orbitals corresponding to $A$ - and $B$-type atoms in order to take into account correct boundary conditions. Consequently, one must reverse the sign of $t_{n n}$, and update the sign of two-body Coulomb matrix elements $\left\langle i j\left|V_{e e}\right| k l\right\rangle$ (note that the sign of $t_{n n n}$ is not affected by the choice of alternating sign basis set).

After diagonalizing the Hartree-Fock Hamiltonian, we obtain Hartree-Fock quasiparticles denoted by the creation operator $b_{p \sigma}^{\dagger}$, with eigenvalues $\epsilon_{p}$ and eigenfunctions $|p\rangle$. We can now fill the new quasiparticle edge states with varying number of electrons and solve the many-body Hamiltonian given by

$$
H=\sum_{p \sigma} \epsilon_{p} b_{p \sigma}^{\dagger} b_{p \sigma}+\frac{1}{2} \sum_{p q r s \sigma \sigma^{\prime}}\left\langle p q\left|V_{e e}\right| r s\right\rangle b_{p \sigma}^{\dagger} b_{q \sigma^{\prime}}^{\dagger} b_{r \sigma^{\prime}} b_{s \sigma} .
$$

The above many-body Hamiltonian can then be diagonalized in subspaces with different total spin projection $S_{z}$ 


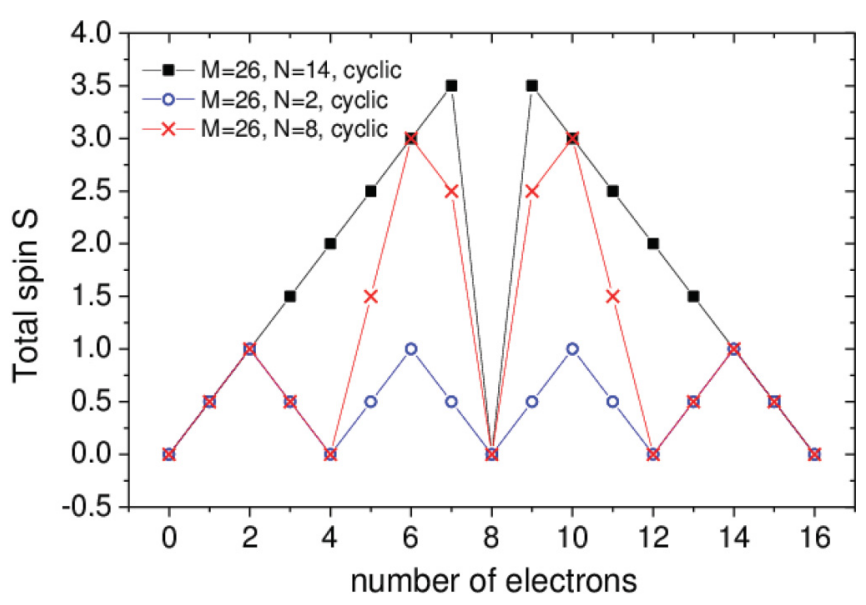

FIG. 7. (Color online) Total spin $S$ as a function of number of electrons occupying the edge states for a cyclic ribbon with length $M=26$ for different widths, $N=2,8$, and 14 .

allowing us to deduce the ground state total spin $S$ of the system for a given number of electrons in the degenerate shell of edge states. Figure 7 show the result obtained for a cyclic ribbon with length $M=26$ for different widths, $N=2,8$, and 14 .

There are eight edge states which can be occupied by up to $N_{e}=16$ electrons. When the system is charge neutral, i.e., $N_{e}=8$, we find that the cyclic ribbon has minimal total spin $S=0$. For the wide ribbon, $N=14$, this result is consistent with infinite length ribbon results where opposite edges are in a antiferromagnetic configuration carrying opposite net spin, with a zero net magnetization. However, here we find that the net magnetization is sensitive to the net charge in the system. If we charge the system with even a single electron or hole, an abrupt change from antiferromagnetic configuration to ferromagnetic configuration occurs. In fact, away from the charge neutrality, the total spin of the edges is maximized. When $N_{e}=0$ or $N_{e}=16$ we have a completely empty or fully occupied edge states, leading to total zero spin again in a paramagnetic configuration. In the other limit of thin ribbon, $N=2$, the edge states are not highly degenerate but form a shell structure with double orbital degeneracy. This leads to a Hund's-like rule, where within each shell the total spin is maximized. As a result, we obtain an oscillating net spin: Every time the number of electrons is a multiple of two we obtain $S=1$. When $N=8$ the system can neither be considered thin nor wide enough to have strongly degenerate edge states. The competition between Hund's rules for double shells and net edge magnetization give rise to rather complex oscillations of the total spin as a function of number of electrons.

In Fig. 8 we show the results of exact diagonalization of the quasiparticle Hamiltonian [Eq. (17)] for the same ribbon as in Fig. 7, but in Möbius configuration. Although both ribbons have the same number of atoms, the zero energy shells are different. For the cyclic case there are $N=8$ degenerate states half-filled at $N_{e}=8$. For a band in Möbius configuration the zero energy shell is split into $N=7$ and $N=2$ subshells. The lowest degenerate subshell is half-filled at $N_{e}=7$ and not at the charge neutrality point $N_{e}=8$. For the wide ribbon $(N=14)$, at half-filling $N_{e}=7$, the system is found to be ferromagnetic. This can be understood

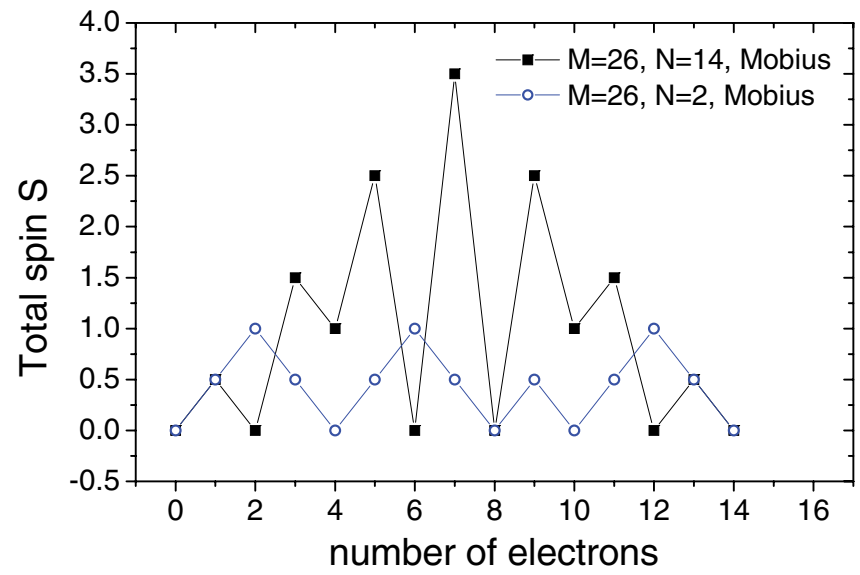

FIG. 8. (Color online) Total spin $S$ as a function of number of electrons occupying the edge states for a Möbius ribbon with length $M=26$ for different widths, $N=2$ and 14 .

from the fact that Möbius configuration is a one edged system, behaving like the zigzag edge of semi-infinite bulk graphene, or of a triangular graphene quantum dot. ${ }^{52-54}$ The magnetization is lost in the charge neutral case $N_{e}=8$. This is in agreement with earlier mean-field Hubbard calculations using s-type orbitals, where opposite edges are found to be in a antiferromagnetic configuration, but with a spin domain wall helping to overcome the magnetic frustration along the zigzag edge of the Möbius strip. This finding is also consistent with spin polarization of a single edge in a triangular graphene quantum dot with sevenfold degenerate shell where addition of an eighth electron leads to full spin depolarization. ${ }^{52}$ Away from charge neutrality, the effects of long range Coulomb interactions become increasingly important and Wigner localization occurs. A detailed study of the effect of Wigner localization and corresponding spin states will be published elsewhere. For the thin Möbius ribbon $N=2$, as for the cyclic ribbon, the degeneracy between edge states is lifted, and shell structure becomes prominent. There is a difference, however, due to the broken electron-hole symmetry in the single particle energy spectrum of the Möbius ribbon. Thus, Hund's rule that maximizes the total spin within a shell still applies, but the total spin spectrum does not have the electron-hole symmetry anymore.

In Fig. 9 we plot the total spin as a function of number of electrons occupying the edge states of odd $N$ Möbius configurations, with $M=25$, for a thin ring $N=3$, and wide ring $N=13$. For the wide ring, at half-filling $N_{e}=8$, the edge states are fully spin polarized, and the polarization is lost if the system is charge neutral at $N_{e}=8$, similar to the even $N$ Möbius ring. However, in contrast with the even $N$ case, the total spin diagram has strongly broken electron-hole symmetry. To understand this, let us assume that in the limit of a very wide ribbon, the edge states almost completely lie on the edge atoms. Those edge atoms of the Möbius strip can be thought of as a single chain ring with an effective hopping between them. For odd $N$, there are an odd number of edge atoms in the chain, leading to a broken electron-hole symmetry. For even $N$, as we have seen in Fig. 8, the electron-hole symmetry is recovered for wide Möbius ribbons. For the 


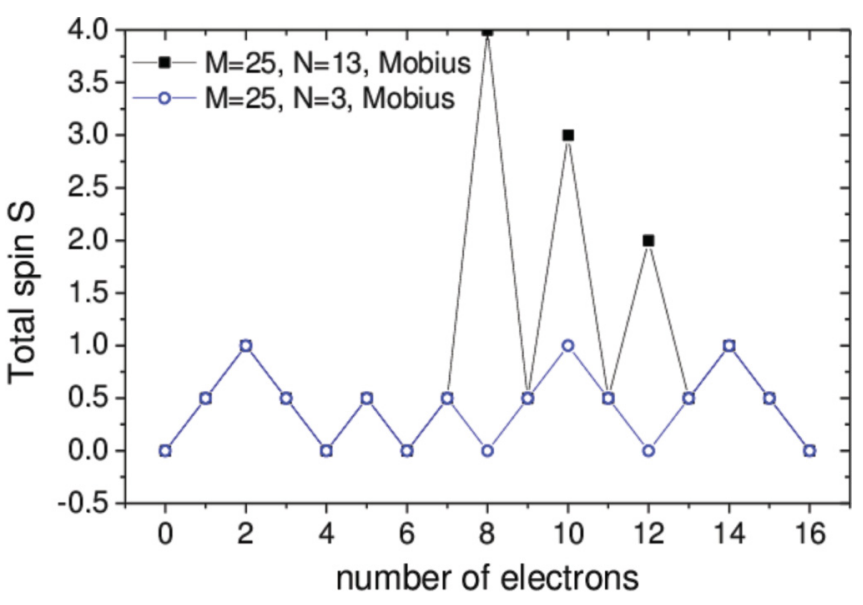

FIG. 9. (Color online) Total spin $S$ as a function of number of electrons occupying the edge states for a Möbius ribbon with length $M=25$ for different widths, $N=3$ and 13 .

$M=25, N=3$ system shown in Fig. 9, once again the degeneracy of the edge states is lost and the spin oscillations follow closely the shell structure, as in the $M=26, N=2$ system shown in Fig. 8.

\section{CONCLUSIONS}

We have presented a theory of the electronic properties of gated graphene nanoribbon rings with zigzag edges in Möbius and cylindrical configurations. The finite width opened a gap and nontrivial topology of the Möbius ring led to a single edge with edge states in the presence of an effective gauge field in analogy to topological insulators. The single zigzag edge led to a shell of degenerate states at the Fermi level and a ferromagnetic (FM) ground state at half-filling due to electron-electron interactions. The half-filling corresponds to a Möbius strip with one electron removed. For a charge neutral Möbius ring the ground state is found to be antiferromagnetic. For fractional filling both the magnetic moment and the energy gap is found to oscillate as a function of the shell filling. In cylindrical rings the two edges lead to AF ground state at half-filling but FM ground state at fractional fillings.

\section{ACKNOWLEDGMENTS}

The authors thank Y. Shen, P. Potasz, and M. Korkusinski for discussions, and NSERC, NRC, Canadian Institute for Advanced Research, and UCCS-CRCW for support.
${ }^{1}$ P. R. Wallace, Phys. Rev. 71, 622 (1947).

${ }^{2}$ A. H. C. Neto, F. Guinea, N. M. R. Peres, K. S. Novoselov, and A. K. Geim, Rev. Mod. Phys. 81, 109 (2009).

${ }^{3}$ K. S. Novoselov, A. K. Geim, S. V. Morozov, D. Jiang, Y. Zhang, S. V. Dubonos, I. V. Grigorieva, and A. A. Firsov, Science 306, 666 (2004).

${ }^{4}$ Y. B. Zhang, Y. W. Tan, and H. L. Stormer, P. Kim, Nature (London) 438, 201 (2005).

${ }^{5}$ M. L. Sadowski, G. Martinez, M. Potemski, C. Berger, and W. A. de Heer, Phys. Rev. Lett. 97, 266405 (2006).

${ }^{6}$ C. L. Kane and E. J. Mele, Phys. Rev. Lett. 95, 226801 (2005).

${ }^{7}$ L. Brey and H. A. Fertig, Phys. Rev. B 73, 195408 (2006).

${ }^{8}$ L. Brey and H. A. Fertig, Phys. Rev. B 73, 235411 (2006).

${ }^{9}$ Y. Yao, F. Ye, X.-L. Qi, S.-C. Zhang, and Z. Fang, Phys. Rev. B 75, 041401(R) (2007).

${ }^{10}$ S. Konschuh, M. Gmitra, and J. Fabian, Phys. Rev. B 82, 245412 (2010).

${ }^{11}$ W. Yao, S. A. Yang, and Q. Niu, Phys. Rev. Lett. 102, 096801 (2009).

${ }^{12}$ D. Gosálbez-Martínez, J. J. Palacios, and J. Fernández-Rossier, Phys. Rev. B 83, 115436 (2011).

${ }^{13}$ Y.-W. Son, M. L. Cohen and S. G. Louie, Nature (London) 444, 347 (2006).

${ }^{14}$ M. Ezawa, Phys. Rev. B 73, 045432 (2006).

${ }^{15}$ S. Ihnatsenka and G. Kirczenow, Phys. Rev. B 83, 245442 (2011).

${ }^{16}$ M. Wimmer, I. Adagideli, S. Berber, D. Tomanek, and K. Richter, Phys. Rev. Lett. 100, 177207 (2008).

${ }^{17}$ O. V. Yazyev and M. I. Katsnelson, Phys. Rev. Lett. 100, 047209 (2008).

${ }^{18}$ L. Yang, M. L. Cohen, and S. G. Louie, Phys. Rev. Lett. 101, 186401 (2008).

${ }^{19}$ J. Jung and A. H. MacDonald, Phys. Rev. B 79, 235433 (2009).
${ }^{20}$ F. Munoz-Rojas, J. Fernandez-Rossier, and J. J. Palacios, Phys. Rev. Lett. 102, 136810 (2009).

${ }^{21}$ J. Jung and A. H. MacDonald, Phys. Rev. B 81, 195408 (2010).

${ }^{22}$ D. Soriano and J. Fernandez-Rossier, Phys. Rev. B 82, 161302(R) (2010).

${ }^{23}$ H. Feldner, Z. Y. Meng, T. C. Lang, F. F. Assaad, S. Wessel, and A. Honecker, Phys. Rev. Lett. 106, 226401 (2011).

${ }^{24}$ T. Hikihara, X. Hu, H.-H. Lin, C.-Yu. Mou, Phys. Rev. B 68, 035432 (2003).

${ }^{25}$ B. Wunsch, T. Stauber, F. Sols, and F. Guinea, Phys. Rev. Lett. 101, 036803 (2008).

${ }^{26}$ S. Dutta, S. Lakshmi, and S. K. Pati, Phys. Rev. B 77, 073412 (2008).

${ }^{27}$ H. Wang and V. W. Scarola, Phys. Rev. B 85, 075438 (2012).

${ }^{28}$ S. Dutta and K. Wakabayashi, Sci. Rep. 2, 519 (2012).

${ }^{29}$ M. Y. Han, B. Ozyilmaz, Y. Zhang, and P. Kim, Phys. Rev. Lett 98, 206805 (2007).

${ }^{30}$ X. Li, X. Wang, L. Zhang, S. Lee, and H. Dai, Science 319, 1229 (2008).

${ }^{31}$ K. A. Ritter and J. W. Lyding, Nat Mater. 8, 235 (2009).

${ }^{32}$ X. Jia, M. Hofmann, V. Meunier, B. G. Sumpter, J. CamposDelgado, J. M. Romo-Herrera, H. Son, Y.-P. Hsieh, A. Reina, J. Kong, M. Terrones, and M. S. Dresselhaus, Science 323, 1701 (2009).

${ }^{33}$ L. Jiao, L. Zhang, X. Wang, G. Diankov, and H. Dai, Nature (London) 458, 877 (2009).

${ }^{34}$ J. Cai, P. Ruffieux, R. Jaafar, M. Bieri, T. Braun, S. Blankenburg, M. Muoth, A. P. Seitsonen, M. Saleh, X. Feng, K. Mllen and R. Fasel, Nature (London) 466, 470 (2010).

${ }^{35}$ C. Tao, L. Jiao, O. V. Yazyev, Y.-C. Chen, J. Feng, X. Zhang, R. B. Capaz, J. M. Tour, A. Zettl, S. G. Louie, H. Dai, and M. F. Crommie, Nat. Phys. 7, 616 (2011). 
${ }^{36}$ A. Chuvilin, E. Bichoutskaia, M. C. Gimenez-Lopez, T. W. Chamberlain, G. A. Rance, N. Kuganathan, J. Biskupek, U. Kaiser, and A. N. Khlobystov, Nat. Mater. 10, 687 (2011).

${ }^{37}$ K. Sasaki, R. Saito, M. S. Dresselhaus, K. Wakabayashi, and T. Enoki, New J. Phys. 12, 103015 (2010).

${ }^{38}$ K. R. Subbaswamy and M. Grabowski, Phys. Rev. B 24, 2168 (1981).

${ }^{39}$ J. E. Hirsch and M. Grabowski, Phys. Rev. Lett. 52, 1713 (1984).

${ }^{40}$ R. Herges, Chem. Rev. 106, 4820 (2006).

${ }^{41}$ D. Ajami and O. Oeckler, A. Simon, and R. Herges, Nature (London) 426, 819 (2003).

${ }^{42}$ K. Wakabayashi and K. Harigaya, J. Phys. Soc. Jpn. 72, 998 (2003).

${ }^{43} \mathrm{~K}$ Harigaya, A Yamashiro, Y Shimoi, and K Wakabayashi, Synth. Met. 152, 261 (2005).

${ }^{44}$ D. Jiang and S. Dai, J. Phys. Chem. C 112, 5348 (2008).

${ }^{45}$ E. W. S. Caetano, V. N. Freire, S. G. dos Santos, D. S. Galvao, and F. Sato, J. Chem. Phys. 128, 164719 (2008).

${ }^{46}$ Z. L. Guo, Z. R. Gong, H. Dong, and C. P. Sun, Phys. Rev. B 80, 195310 (2009).

${ }^{47}$ X. Wang, X. Zheng, M. Ni, L. Zou, and Z. Zeng, Appl. Phys. Lett. 97, 123103 (2010).

${ }^{48}$ E. Heilbronner, Tetrahedron Lett. 29, 1923 (1964).

${ }^{49}$ P. W. Fowler, Phys. Chem. Chem. Phys. 4, 2878 (2002).

${ }^{50}$ J. M. André, B. Champagne, E. A. Perpète, and M. Guillaume, Int. J. Quantum Chem. 84, 607 (2001).
${ }^{51}$ J. E. Hirsch, Phys. Lett. A 374, 3777 (2010).

${ }^{52}$ A. D. Güçlü, P. Potasz, O. Voznyy, M. Korkusinski, and P. Hawrylak, Phys. Rev. Lett. 103, 246805 (2009).

${ }^{53}$ A. D. Güçlü, P. Potasz, and P. Hawrylak, Phys. Rev. B 82, 155445 (2010).

${ }^{54}$ P. Potasz, A. D. Güçlü, A. Wojs, and P. Hawrylak, Phys. Rev. B 85, 075431 (2012).

${ }^{55}$ P. Koskinen, S. Malola, and H. Hakkinen, Phys. Rev. Lett. 101, 115502 (2008).

${ }^{56}$ T. Wassmann, A. P. Seitsonen, A. M. Saitta, M. Lazzeri, and F. Mauri, Phys. Rev. Lett. 101, 096402 (2008).

${ }^{57}$ P. Koskinen, S. Malola, and H. Hakkinen, Phys. Rev. B 80, 073401 (2009).

${ }^{58}$ P. Rakyta, A. Kormányos, J. Cserti, and P. Koskinen, Phys. Rev. B 81, 115411 (2010).

${ }^{59}$ Y. Y. Sun, W. Y. Ruan, X. Gao, J. Bang, Y.-H. Kim, K. Lee, D. West, X. Liu, T.-L. Chan, M. Y. Chou, and S. B. Zhang, Phys. Rev. B 85, 195464 (2012).

${ }^{60}$ O. Voznyy, A. D. Güçlü, P. Potasz, and P. Hawrylak, Phys. Rev. B 83, 165417 (2011).

${ }^{61}$ X. Zhang, O. V. Yazyev, J. Feng, L. Xie, C. Tao, Y.-C. Chen, L. Jiao, Z. Pedramrazi, A. Zettl, S. G. Louie, H. Dai, and M. F. Crommie, ACS Nano 7, 198 (2013). 